\title{
Hemostasia e incisão do pedículo ovariano de gatas submetidas à ovariossalpingo- histerectomia eletiva por meio da técnica das três pinças ou do eletrobisturi bipolar. Estudo comparativo
}

[Hemostasis and incision into the ovary pedicle of cats undergone elective ovariosalpingohysterectomy using the technique of the three bipolar forceps or eletrobisturi. Comparative study]

\author{
E.A. Tudury ${ }^{1}$, A.C.M. Almeida ${ }^{2}$, T.H.T.F. Tavares ${ }^{1 *}$, K.M.H.A.S. Fontes ${ }^{3}$, D.C. Borges ${ }^{3}$ \\ ${ }^{1}$ Universidade Federal Rural de Pernambuco \\ ${ }^{2}$ Profissional autônomo
}

\begin{abstract}
RESUMO
$\mathrm{Na}$ cirurgia de ovariossalpingo-histerectomia $(\mathrm{OSH})$, há várias técnicas para realização da hemostasia e da secção do pedículo ovariano, sendo mais comum a técnica das três pinças. O eletrobisturi bipolar pode ser utilizado com eficácia para hemostasia e corte de tecidos. Objetivou-se nesta pesquisa comparar, em gatas, a hemostasia e a secção do pedículo ovariano mediante o uso do eletrobisturi bipolar com a técnica das três pinças, ambas via celiotomia mediana, em relação ao tempo cirúrgico e à eficiência hemostática (hematócrito). Quarenta gatas, com idade de seis meses a sete anos, peso entre 1,68 e 4,14kg, foram submetidas à hemostasia e à secção desse pedículo, sendo 20 pela técnica convencional e 20 por meio do eletrobisturi bipolar. $\mathrm{Na}$ análise estatística, observou-se que o uso do eletrobisturi bipolar gera uma diminuição média significativa na duração da cirurgia, quando comparado com o outro método, sem diferenças quanto à comparação de hematócritos pré e pós-cirúrgicos. Por meio do modelo de regressão múltiplo, verificou-se que as variáveis: idade, peso, número de gestações, número de partos e número de cios não influenciaram significativamente no tempo. Concluiu-se, portanto, que o eletrobisturi bipolar oferece eficiência e maior rapidez na realização da hemostasia e da diérese do pedículo ovariano de gatas submetidas à OSH eletiva.
\end{abstract}

Palavras-chave: OSH, cirurgia eletiva, hemostasia

\begin{abstract}
In ovariosalpingohisterectomy $(\mathrm{OSH})$ surgery, there are several techniques for hemostasis and resection of ovarian pedicle, the most common being the three tweezers technique. The bipolar eletrobisturi can be used effectively for hemostasis and cutting tissue. The aim of this research was to compare, in cats, hemostasis and resection of ovarian pedicle eletrobisturi with bipolar forceps and the three techniques, both via median celiotomy, in relation to time and surgical hemostatic efficiency (hematocrit). Forty cats aged six months to seven years, weighing between 1.68 and $4.14 \mathrm{~kg}$, underwent hemostasis and pedicle of this section, 20 with the conventional technique and 20 with eletrobisturi bipolar. Statistical analysis showed that the use of bipolar eletrobisturi generates a significant decrease in the mean duration of surgery when compared with a method without differences in hematocrit comparing pre-and postsurgery. Through the multiple regression model we found that the variables age, weight, number of pregnancies, number of births and number of cycles did not significantly influence the time. We conclude, therefore, that the bipolar eletrobisturi is efficient and fast for hemostasis and dieresis ovarian pedicle of cats undergoing elective ovariohysterectomy.
\end{abstract}

Keywords: OSH, elective surgery, coagulation

Recebido em 4 de junho de 2013

Aceito em 19 de fevereiro de 2014

*Autor para correspondência (corresponding author)

E-mail: thaizavet@gmail.com 


\section{INTRODUÇÃO}

A ocorrência de hemorragias é a causa mais comum de morte depois de uma ovariossalpingohisterectomia $(\mathrm{OSH})$ e pode ser causada pela ruptura dos vasos ovarianos quando o ligamento suspensor sofre estiramento, ou durante a secção dos vasos uterinos (Birchard e Sherding, 2003; Santos et al., 2009).

Em videolaparoscopia utiliza-se a eletrocoagulação para hemostasia de vasos. Segundo estudo feito por Schiochet et al. (2007), em que foram realizadas OSH laparoscópica em felinos, comparando-se métodos diferentes de hemostasia (eletrocauterização bipolar, clipes de titânio e ligadura com fio de sutura), obteve-se como resultado uma excelente oclusão dos vasos proporcionada pelo eletrocautério bipolar.

Bart et al. (2003) compararam o eletrocautério monopolar e o bipolar na ovariectomia laparoscópica em cães e relataram que o último proporcionou redução do tempo cirúrgico e menor hemorragia durante o procedimento. Schiochet et al. (2007) afirmam que a diatermia bipolar representa um avanço em relação à monopolar, pois diminui os efeitos indesejáveis desta e possibilita maior controle sobre as lesões causadas no tecido alvo.

Objetivou-se nesta pesquisa comparar, no transcorrer de OSH eletiva de gatas, a hemostasia e a secção do pedículo ovariano mediante o uso do eletrobisturi bipolar com a técnica das três pinças, via celiotomia mediana, em relação ao tempo cirúrgico e à eficiência hemostática, assim como avaliar a influência da idade, do peso, do número de gestações, de partos e de cios em relação ao tempo cirúrgico obtido em cada método.

\section{MATERIAL E MÉTODO}

Neste trabalho, foram castradas 40 gatas, com idade variando de seis meses a sete anos, peso médio de $2,77 \mathrm{~kg}(1,68$ a $4,14 \mathrm{~kg})$, sem raça definida ou siamês, atendidas no Hospital Veterinário (HV) da Universidade Federal Rural de Pernambuco (UFRPE), conforme aprovação da comissão de ética do Departamento de Medicina Veterinária dessa instituição (Número do protocolo: 23082.013399/2008).
Das gatas submetidas ao procedimento cirúrgico de OSH eletiva, para hemostasia e secção do pedículo ovariano, em 20 (50\%) (média: $2,64 \mathrm{~kg}$ ) foi utilizada a técnica das três pinças, e em 20 $(50 \%)$ (média: $2,91 \mathrm{~kg}$ ) apenas o eletrobisturi bipolar. As cirurgias foram realizadas na sequência: OSH tradicional - OSH eletrobisturi bipolar/OSH eletrobisturi bipolar - OSH tradicional e, assim, sucessivamente, com distribuição aleatória das gatas em cada grupo.

Cada paciente foi submetido à anamnese e a exame físico detalhado, os quais visaram obter informações relativas à possibilidade da presença de enfermidade, assim como ao número de gestações, ao parto e aos cios anteriores ao experimento. Gatas prenhes, no cio ou puerpério, desidratadas, ou com menos de seis meses, foram descartadas. No dia da cirurgia, os pacientes foram submetidos à nova avaliação física do volume globular, dos tempos de sangria e de coagulação e, então, encaminhados à cirurgia.

A determinação dos volumes globulares iniciou-se pela retirada de sangue da veia cefálica e imediato preenchimento de tubo de micro-hematócrito de $75 \mathrm{~mm}$ contendo heparina, sendo este centrifugado (Centrífuga para micro-hematócrito - Gemmy Industrial Corp, modelo KHT - 400) a 10000rpm durante cinco minutos para, depois, ser realizada em tabela a leitura da porcentagem correspondente ao volume globular. Os dados foram transcritos para um quadro contendo nome do paciente, valor do hematócrito 48 horas antes e após a cirurgia.

Todos os procedimentos cirúrgicos foram realizados pelo mesmo cirurgião. $\mathrm{O}$ procedimento cirúrgico foi cronometrado desde o início da procura dos ovários e do útero até a completa retirada deles da cavidade abdominal.

Pela técnica de ovariossalpingo-histerectomia convencional, a incisão iniciou aproximadamente $1 \mathrm{~cm}$ caudal ao umbigo e foi estendida cerca de $3 \mathrm{~cm}$. Localizou-se o corno uterino com o dedo indicador e rompeu-se o ligamento suspensor do ovário, com uma tensão caudal ao longo da parede corporal dorsal. A seguir, fez-se uma janela no mesovário, caudal ao complexo AV ovariano, pela qual se pinçou o pedículo (AV) ovariano com três pinças hemostáticas (tipo crile, 
$16 \mathrm{~cm}$ de comprimento), que foi resseccionado entre a primeira (mais ventral) e a segunda pinça.

Utilizou-se um fio absorvível (categute cromado 2-0) para todas as ligaduras. Aplicou-se uma ligadura ao redor do pedículo, distal à terceira pinça (mais dorsal), que foi removida à medida que se apertou a ligadura, de forma que ela repousasse no sulco de tecido esmagado criado por essa pinça (Fig. 1).

Prendeu-se o pedículo com uma pinça de polegar e liberou-se a pinça remanescente. Inspecionouse se o pedículo estava sangrando. Não havendo sangramento, recolocou-se o coto pelicular na cavidade abdominal. Foi feita a exteriorização do outro corno uterino, e, então, repetiu-se o procedimento de ovariectomia.
A seguir, exteriorizou-se o corpo uterino, localizou-se a cérvix, onde foram colocadas três pinças hemostáticas (tipo Crile, $16 \mathrm{~cm}$ de cumprimento), seccionou-se o corpo uterino entre as pinças mais craniais e colocou-se uma ligadura transfixada em figura de 8 caudal à terceira pinça, que foi removida antes do aperto dos nós. Avaliou-se se o coto uterino estava sangrando e, não havendo sangramento, este foi recolocado na cavidade.

A parede abdominal foi fechada em três camadas com os seguintes respectivos fios e padrões de sutura: fáscia/ linha alba (categute cromado 2-0, isolado simples), tecido subcutâneo (categute cromado 3-0, ziguezague) e pele ( náilon 3-0, isolado simples).

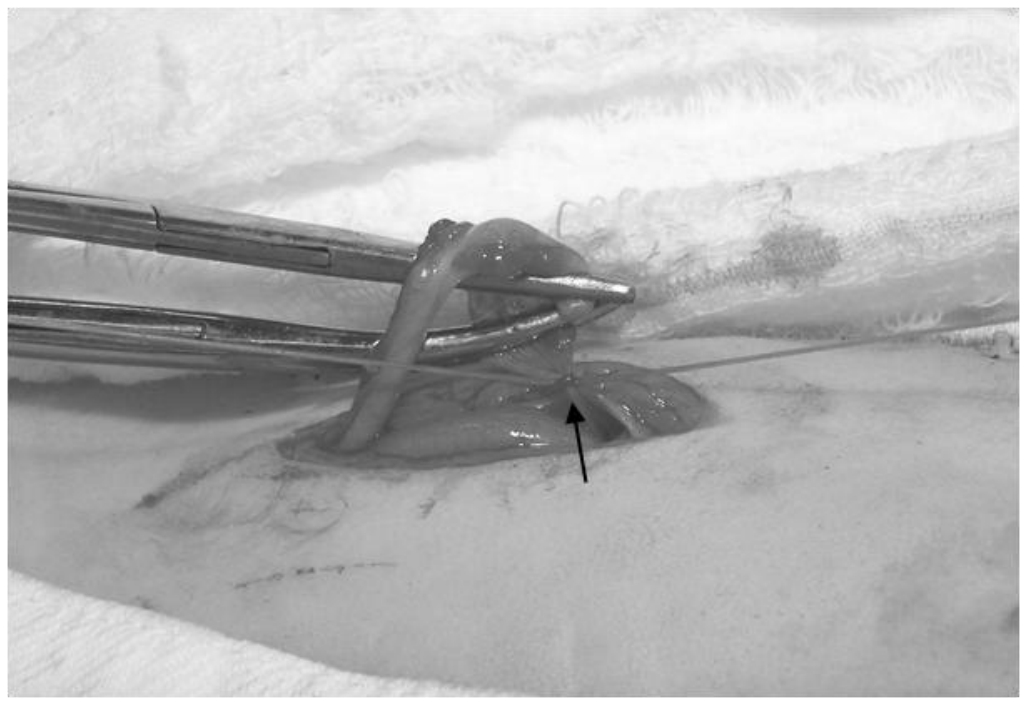

Figura 1. Sutura circular feita com categute cromado 2-0 estrangulando os vasos do pedículo ovariano (seta preta) repousando no sulco criado no tecido esmagado pela pinça mais dorsal, já removida, em gatas submetidas à OSH convencional.

Já pela técnica de ovariectomia com eletrobisturi bipolar, a incisão abdominal também foi realizada a aproximadamente $1 \mathrm{~cm}$ do umbigo e se estendeu cerca de $3 \mathrm{~cm}$ caudalmente. Após localizar o corno uterino com o dedo indicador, esticou-se e prendeu-se o ligamento suspensor com uma pinça hemostática tipo crile, onde foi feita uma janela no mesovário caudal ao complexo AV ovariano.

Com a pinça do eletrobisturi bipolar (Bisturi Cirúrgico Eletrônico 150watts Mono e Bipolar BP150 Emai), em potência de 40W e acionado no efeito corte e hemostasia, procedeu-se à incisão do complexo arteriovenoso ovariano, o qual foi seccionado junto ao mesovário (Fig. $2 \mathrm{~A}$ e B). Após observar se o pedículo estava sangrando, removeu-se e liberou-se a pinça. Não havendo sangramento, recolocou-se o coto pedícular na cavidade abdominal e, então, repetiu-se o procedimento da hemostasia e da secção do pedículo ovariano contralateral.

A partir da exteriorização do corpo uterino, o procedimento igualava-se àquele que foi realizado no grupo da técnica convencional. 


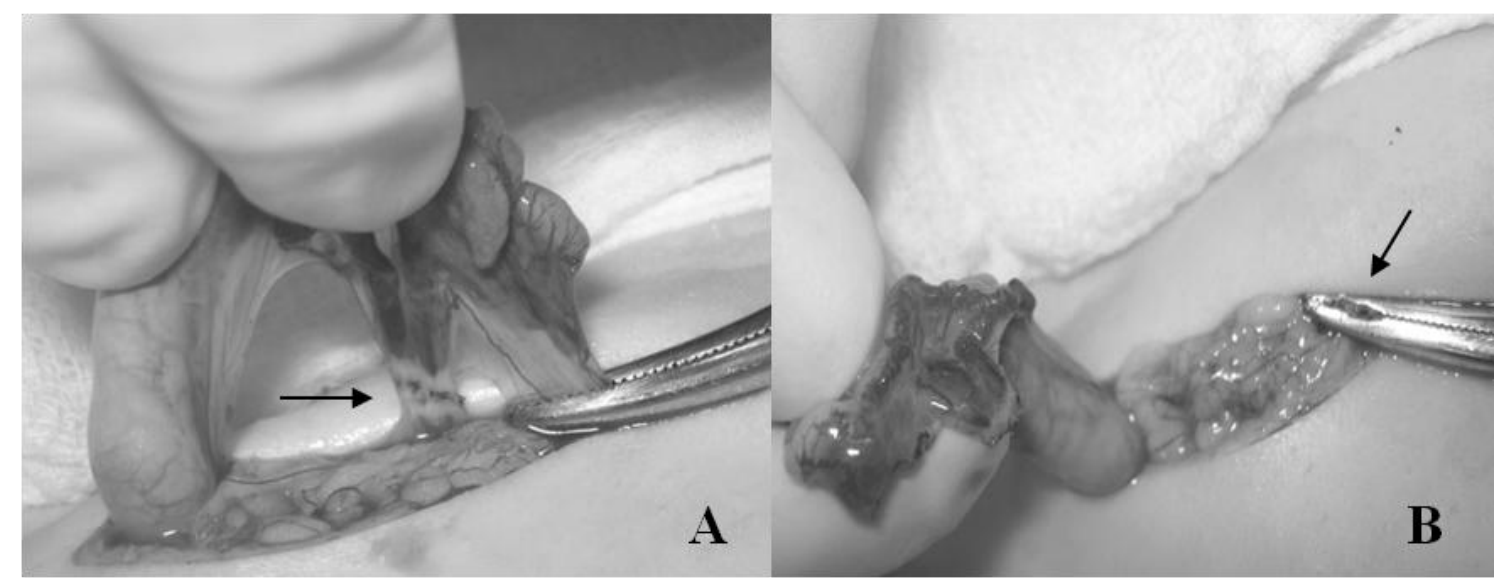

Figura 2. A. Hemostasia do complexo AV no pedículo ovariano com eletrobisturi bipolar - notar aspecto tecidual esbranquiçado decorrente da cauterização (seta preta); B. pedículo AV ovariano já seccionado, evidenciando ausência de sangramento advindo da artéria ovárica (seta preta), em gatas submetidas à OSH com eletrobisturi bipolar.

Quarenta e oito horas após a cirurgia, os pacientes retornaram para avaliação clínica e exame de micro-hematócrito. No sétimo dia de pós-operatório, foi feita nova avaliação do estado geral do paciente, e a ferida cirúrgica foi avaliada quanto a sinais de deiscência, infecção e edema, assim como a cicatrização; em seguida, efetuouse a remoção dos pontos cutâneos.

A análise descritiva dos dados foi feita por meio de tabelas e/ou gráficos para as variáveis qualitativas, bem como das medidas de tendência central e de dispersão (mínimo, máximo, média, mediana e desvio-padrão) para as variáveis quantitativas. Para comparação de média, foi utilizado o teste t de Student, entretanto, antes de se fazer a comparação entre as médias, foi realizado o teste de Kolmogorov-Smirnov, a fim de verificar a satisfação da condição de normalidade. Além do teste de comparação de médias, utilizou-se a teoria dos modelos lineares múltiplos, com o objetivo de verificar se o peso, a idade, o número de gestações, o número de partos e o número de cios influenciam significativamente no tempo cirúrgico nos animais. Utilizou-se o nível de significância de $5 \%$ em todos os testes realizados.

\section{RESULTADOS E DISCUSSÃO}

Os exames complementares tempo de sangria e de coagulação foram realizados com o intuito de se avaliarem pré-operatoriamente os animais quanto à possibilidade de haver hemorragia trans e pós-operatória que gerasse interpretações erradas no que se refere à eficiência hemostática das técnicas utilizadas, observando-se que todos os animais apresentavam-se dentro dos valores normais (tempo de coagulação e tempo de sangria normal de um a cinco minutos), para cada teste realizado, segundo Kaneco (1989) e Marques et al. (2009).

Preferiu-se a avaliação do hematócrito à contagem total de hemácia (CTH) e à concentração de hemoglobina do sangue total (Hb), uma vez que os dois últimos são mais sujeitos a erro e variam quase exatamente em paralelo com o hematócrito (Schalm, 1961; Kerr, 2003). Essa avaliação foi realizada em dois momentos: dois dias antes, para que não houvesse influência do jejum e do estresse précirúrgico, pois estes aumentam o valor do hematócrito, sendo o último devido à contração esplênica com liberação das hemácias na circulação (Coles, 1984); e dois dias após, para que se pudesse avaliar hemorragia pósoperatória, já que, em casos de hemorragia aguda, a perda de sangue imediata resulta em pouca ou nenhuma alteração no hematócrito, em razão da perda simultânea de hemácias e fluidos plasmáticos. Durante as horas seguintes, quando o volume plasmático for restabelecido por líquido advindo do espaço extravascular, o hematócrito diminuirá (Birchard e Sherding, 2003; Fossum, 2008). 
Para a ovariectomia de três animais (15\%) (cujos vasos ovarianos possuíam o mesmo calibre das outras gatas), foram realizados dois procedimentos: inicialmente, fez-se uso do eletrobisturi bipolar, com sucesso, em um pedículo ovariano, mas, como houve sangramento considerável no segundo pedículo, procedeu-se à realização da ligadura com o uso de fio de categute 2-0, fato relatado por Silva et al. (2012), o que discorda dos resultados de Schiochet et al. (2007), que, em pesquisa, compararam diversos métodos de hemostasia e constataram que, na ovariectomia, o eletrobisturi bipolar não ocasionou hemorragia, pois efetuaram nas suas cirurgias três pontos de eletrocauterização ao longo do pedículo ovariano, ficando duas no pedículo remanescente e seccionando-se na mais dorsal. Acredita-se que a hemorragia causada nesses animais foi devido à limpeza inadequada das pontas da pinça do eletrobisturi, o que retardou a passagem do estímulo elétrico e ocasionou o corte com a hemostasia inapropriada, o que está de acordo com com Fossum (2008). Apesar desses três casos, não houve óbito em $100 \%$ das gatas submetidas à ovariossalpingo-histerectomia decorrente de hemorragia, uma das complicações pós-operatórias citadas por Slatter (2007) e Schiochet et al. (2007).

Como citado por Fossum (2008), a eletrocoagulação realizada nos demais pedículos ocorreu de forma satisfatória, como uma mancha esbranquiçada surgindo na superfície do tecido, não havendo, nesses casos, a ocorrência de hemorragia após a secção nesse mesmo local.

A incisão realizada pode ser considerada minimamente invasiva $(3 \mathrm{~cm})$, mas, nos três $(7,5 \%)$ casos em que houve hemorragia, foi necessário estender a incisão para melhor visualização das estruturas, conforme recomendado por Chioratto e Tudury (2008).

Não foi observada dificuldade em relação à exteriorização dos ovários devido ao ligamento suspensor, o que está em discordância de Dyce et al. (1997) e Stone et al. (1998), quando citam que o ligamento suspensor dificulta a exteriorização do ovário.
Todos os animais foram submetidos à administração de analgésico (tramadol) no préoperatório, e não se observou quadro de dor no transcirúrgico (pinçamento, coagulação e secção) nem no pós-operatório, bem como não houve diferença entre a técnica tradicional e a com eletrobisturi.

Baldwin et al. (1998) e Brun (2009) citam que o eletrobisturi pode ser usado para controlar hemorragias em artérias $\leq 1 \mathrm{~mm}$ e veias $\leq 2 \mathrm{~mm}$ de diâmetro, o que foi observado no presente estudo, já que os calibres dos vasos encontravam-se dentro desses valores.

Considera-se que o uso do eletrobisturi possui como vantagem sua simplicidade de aplicação, a redução do tempo cirúrgico e, consequentemente, do tempo anestésico, o que torna a cirurgia mais dinâmica e possibilita a realização de um número maior de cirurgias, além de não deixar na cavidade nenhum material estranho ao organismo quando se compara com o uso de fios ou aplicação de clips. A experiência evidenciou que esta é uma técnica mais fácil de realização quando comparada à técnica das três pinças, segundo descrito por Baldwin et al. (1998), Lazzeri (1994) e Fuller (2000). Com o mesmo intuito de evitar ligaduras, hemorragias e retardamento do procedimento, Vicente et al. (2013) realizaram com sucesso a hemostasia do pedículo ovariano, utilizando só torção vascular.

$\mathrm{Na}$ análise estatística dos tempos de execução, foram utilizados apenas 17 animais no grupo eletrobisturi, pois três tiveram a cirurgia revertida para convencional devido ao sangramento.

Ao se comparar o método convencional com o eletrobisturi bipolar em relação à média do tempo (procura do ovário e do útero até a completa retirada destes da cavidade abdominal), verificou-se que o eletrobisturi bipolar apresenta aproximadamente 290,8 segundos (quatro minutos e 85 segundos) a menos que o método convencional (p-valor $<0,001$ ) (Tab. 1). 


\section{Tudury et al.}

Tabela 1. Análise descritiva e comparação de médias da variável: tempo de procura do ovário e do útero até a completa retirada destes órgãos da cavidade abdominal, entre o método convencional e com o uso do eletrobisturi

\begin{tabular}{lcccccccc} 
Variável & N & Mínimo & Máximo & Mediana & Média & $\begin{array}{c}\text { Desvio- } \\
\text { padrão }\end{array}$ & $\begin{array}{c}\text { IC (intervalo } \\
\text { de confiança) } \\
(95 \%)\end{array}$ & p-valor \\
\hline Tempo & & & & & & & & \\
Clássica & 20 & 240 & 1200 & 782,5 & 711,30 & 218,9 & $608,9-813,8$ & $<0,001$ \\
Eletrobisturi & 17 & 240 & 663 & 418 & 420,5 & 125,7 & $355,9-485,1$ & \\
\hline
\end{tabular}

Tempo - Tempo da elevação do útero e do ovário até a ressecção (em segundos).

*p-valor do teste t de Student para comparação de médias.

$\mathrm{Na}$ análise estatística dos hématócritos, foram utilizados 19 animais no grupo eletrobisturi, já que em um obteve-se um hematócrito muito abaixo dos valores normais, sendo constatada, posteriormente, falha na realização do exame.

Na Tab. 2 e na Fig. 3, observa-se que o percentual médio de hematócritos pelo método convencional e pelo uso do eletrobisturi não diferiu, estatisticamente, tanto dois dias antes da cirurgia ( $\mathrm{p}$-valor $=0,988$ ) quanto dois dias depois da cirurgia $(p$-valor $=0,834)$. Ainda, nota-se que as medianas HT1 e HT2, tanto no método convencional quanto no método eletrobisturi, são próximas da média, o que mostra que o teste de comparação de média possui conclusões fidedignas (Morettin e Bussad, 2002; Moore, 2005).

Tabela 2. Análise descritiva e comparação de médias das variáveis valor do hematócrito dois dias antes da cirurgia e valor do hematócrito dois dias após a cirurgia, entre o método convencional e com o uso do eletrobisturi

\begin{tabular}{lcccccccc}
\hline Variável & N & Mínimo & Máximo & Mediana & Média & $\begin{array}{l}\text { Desvio- } \\
\text { padrão }\end{array}$ & IC(95\%) & $\begin{array}{l}\text { p- } \\
\text { valor }\end{array}$ \\
\hline HT1 & & & & & & & & \\
Clássica & 20 & 0,25 & 0,40 & 0,310 & 0,325 & 0,052 & $0,301-0,349$ & 0,988 \\
Eletrobisturi & 19 & 0,21 & 0,45 & 0,320 & 0,325 & 0,057 & $0,298-0,353$ & \\
\hline HT2 & & & & & & & & \\
Clássica & 20 & 0,23 & 0,45 & 0,32 & 0,324 & 0,061 & $0,296-0,352$ & 0,834 \\
Eletrobisturi & 19 & 0,21 & 0,39 & 0,35 & 0,328 & 0,054 & $0,302-0,354$ & 0,834 \\
\hline
\end{tabular}

HT1 - Valor do hematócrito dois dias antes da cirurgia.

HT2 - Valor do hematócrito dois dias após a cirurgia.

*p-valor do teste t de Student para comparação de médias.

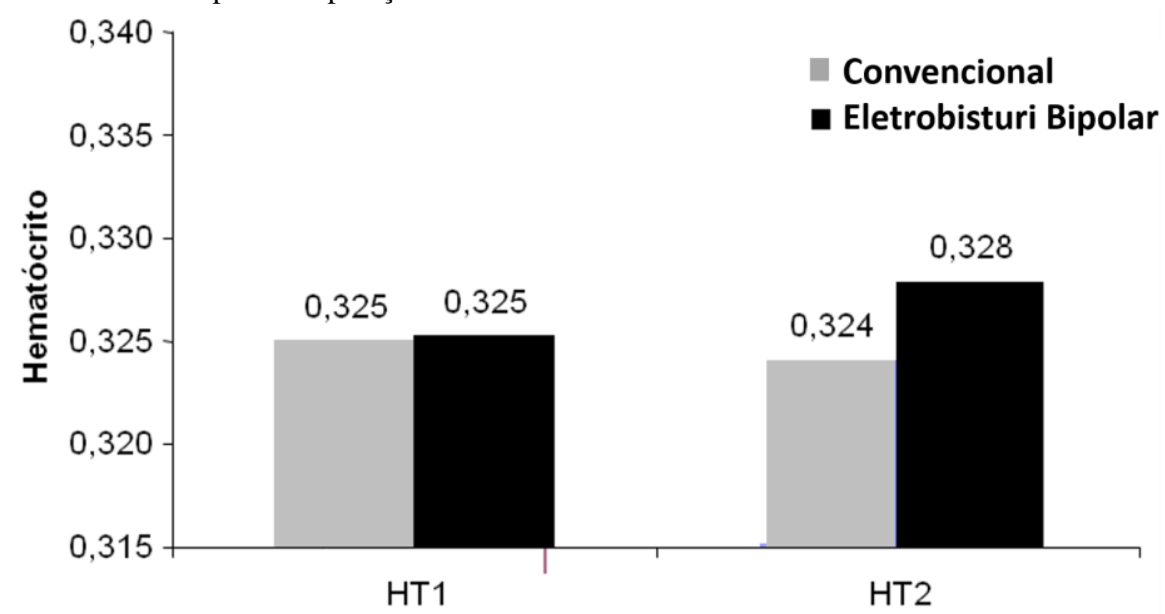

Figura 3. Comparação da média de hematócrito dois dias antes (HT1) e dois dias após (HT2) a cirurgia entre as técnicas de OSH convencional e com o eletrobisturi bipolar. 
Por meio do modelo de regressão múltipla, verificou-se que as variáveis: idade, peso, número de gestações, número de partos e número de cios não influenciam significativamente no tempo de procura do ovário e do útero até a completa retirada desses orgãos da cavidade abdominal. Além disso, confirmou-se que o método utilizado na OSH influencia, estatisticamente, no tempo, uma vez que apenas esta variável ficou nos modelos estimados ( $p$ valor <0,001) (vide Tab. 3).

Tabela 3. Análise de modelos de regressão múltipla do tempo de procura do ovário e do útero até a completa retirada desses órgãos da cavidade abdominal com a idade, peso, gestação, parto, cio e material usado na OSH

\begin{tabular}{lcccc}
\hline \multicolumn{1}{c}{ Variável } & Valor estimado & Erro-padrão & t-valor & $*$ p-valor \\
\hline Intercepto & 711,30 & 42,43 & 16,76 & $<0,001$ \\
OSH [eletrobisturi] & $-249,75$ & 60,00 & $-4,16$ & $<0,001$ \\
\hline *p-valor do teste t de Student & & & &
\end{tabular}

\section{CONCLUSÃO}

Conclui-se que o eletrobisturi bipolar é eficiente na hemostasia e na secção dos pedículos ovarianos de gatas submetidas à OSH eletiva, pois essa técnica resulta em menor tempo cirúrgico, quando comparada ao método convencional, sem diferenças quanto à comparação de hematócritos pré e póscirúrgicos, tempo esse não influenciado pela idade, pelo peso, pelo número de gestações, de partos e de cios.

\section{AGRADECIMENTOS}

Ao CNPq, pela bolsa de mestrado; ao Laboratório Ourofino, pelos medicamentos cedidos.

\section{REFERÊNCIAS}

BALDWIN, C.J.; COWELL, R.L.; KOSTOLICH, M.; TYLER, R.D. Hemostasia: fisiologia e tratamento dos distúrbios hemorrágicos em pacientes cirúrgicos. In: SLATTER, D. Manual de cirurgia de pequenos animais. 2.ed., São Paulo: Manole, 1998. p.36-65.

BART, E.B.J.; GOETHEN, V.; ROSENVELDT, K.W. Monopolar versus bipolar eletrocoagulation in canine laparoscopic ovariectomy. A nonrandomized, prospective clinical trial. Vet. Surg. v.32, p.464-470. 2003.

BIRCHARD, S.J.; SHERDING, R.G. Manual Saunders-Clínica de pequenos animais. 2.ed. São Paulo: Roca, 2003. 1783p.
BRUN, M.V. Cirurgia laparoscópica em cães. In: TUDURY, E.A.; POTIER, G.M.A. Tratado de técnica cirúrgica veterinária. São Paulo: MedVet., 2009. p.243-261.

CHIORATTO, R.; TUDURY, E.A. Celiotomia laparotomia. In: TUDURY, E.A.; POTIER, G.M.A. Tratado de técnica cirúrgica veterinária. São Paulo: MedVet, 2009. p.223-242.

COLES, E.H. Patologia clínica veterinária. 3. ed. São Paulo: Manole, 1984. 566p.

DYCE, K.M.; SACK, W.O.; WENSING, C.J.G. Tratado de anatomia veterinária. 2. ed. Rio de Janeiro: Guanabara koogan, 1997. 663p.

FOSSUM, T.W. Cirurgia de pequenos animais. 3.ed. São Paulo: Roca, 2008. 1632p.

FULLER, J.R. Tecnologia cirúrgica - Princípios e prática. 3.ed. Rio de Janeiro: Guanabara Koogan, 2000. 623p.

KANECO, J.J. Clinical biochemistry of domestic animals. 4. ed. California: Academic press INC. 1989. 932p.

KERR, M.G. Exames laboratoriais em medicina veterinária. Bioquímica clínica e hematológica. 2.ed. São Paulo: Roca, 2003. 436p.

LAZZERI, L. Técnica operatória veterinária. Belo Horizonte: Editora Manole LTDA, 1994. $415 \mathrm{p}$.

MARQUES, N.B.; VIEIRA, M.O.A.; TUDURY, E.A. Hemostasia cirúrgica. In: TUDURY, E.A.; POTIER, G.M.A. Tratado de técnica cirúrgica veterinária. São Paulo: Med Vet, 2009. p.45-62.

MOORE, D.S. A estatística básica e sua prática. 3.ed. Rio de Janeiro: LTC, 2005. 658p. 
MORETTIN, P.A.; BUSSAD, N.O. Estatística básica. Rio de Janeiro: Saraiva, 2002. 540p.

SANTOS, F.C.; CORREIA, T.P.; RAHAL, S.C. Complicações da esterilização cirúrgica de fêmeas caninas e felinas. Revisão de literatura. Vet. Zootec. v.16, p.8-18, 2009.

SCHALM, O.W. Veterinary hematology. Califórnia: Academic press INC, 1961. 386p.

SCHIOCHET, F.; BECK, C.A.C.; STELIDE, R. Ovariectomia laparoscópica em uma gata com ovários remanescentes. Acta Sciential Veterinarie, Rio Grande do Sul, v.32, p.245-248, 2007.

SILVA, A.C.; SILVA, C.E.; PELUSO, E.M.; TUDURY, E.A. Esterilização em gatas mediante salpingectomia parcial (incluindo prenhes) versus ovariossalpingohisterectomia. Cienc. Rural, v.42, p.507-513, 2012.
SLATTER, D. Manual de cirurgia de pequenos animais. 3.ed. São Paulo: Manole, 2007. 2806p.

STONE, E.A.; CANTRELL, C.G.; SHARP, N.J. Ovário e útero. In: SLATTER, D. Manual de cirurgia de pequenos animais. Manole: São Paulo, 1998. p.1540-1558.

VICENTE, P.U.C. Técnica de ovário-salpingohisterectomia, em felinos da espécie Felis catus (gato doméstico), sem o uso de fios ou lacres. A Hora Veterinária, 2013. p.40-42. 